\title{
APOLAR SCHEMES OF ALGEBRAIC FORMS
}

\author{
JAYDEEP CHIPALKATTI
}

\begin{abstract}
This is a note on the classical Waring's problem for algebraic forms. Fix integers $(n, d, r, s)$, and let $\Lambda$ be a general $r$-dimensional subspace of degree $d$ homogeneous polynomials in $n+1$ variables. Let $\mathcal{A}$ denote the variety of $s$-sided polar polyhedra of $\Lambda$. We carry out a case-by-case study of the structure of $\mathcal{A}$ for several specific values of $(n, d, r, s)$. In the first batch of examples, $\mathcal{A}$ is shown to be a rational variety. In the second batch, $\mathcal{A}$ is a finite set of which we calculate the cardinality.
\end{abstract}

Mathematics Subject Classification (2000): 14N05, 14 N15.

Keywords: Waring's problem, apolarity, polar polyhedron.

\section{InTRODUCTION}

We begin with a classical example to illustrate the theme of this paper. Let $F_{1}, F_{2}$ be general quadratic forms in variables $x_{0}, \ldots, x_{n}$, with coefficients in $\mathbf{C}$. It is then possible to diagonalize the $F_{i}$ simultaneously (see [17, Ch. 22]), i.e., one can find linear forms $L_{1}, \ldots, L_{n+1}$ such that

$$
F_{i}=c_{i 1} L_{1}^{2}+\cdots+c_{i(n+1)} L_{n+1}^{2},
$$

for $i=1,2$, and some constants $c_{i j} \in \mathbf{C}$. Moreover, up to rescaling there is a unique choice for the set $\left\{L_{1}, \ldots, L_{n+1}\right\}$. This result naturally leads to similar questions about forms of higher degree, where much less is known in general.

Now assume that $F_{1}, \ldots, F_{r}$ are forms of degree $d$ in $x_{0}, \ldots, x_{n}$. Let $Z=\left\{L_{1}, \ldots, L_{s}\right\}$ be a collection of linear forms in the $x_{i}$, such that it is possible to write

$$
F_{i}=c_{i 1} L_{1}^{d}+\cdots+c_{i s} L_{s}^{d}, \quad 1 \leq i \leq r
$$

for some constants $c_{i j} \in \mathbf{C}$. In nineteenth century terminology (introduced by Reye), $Z$ is then called a polar $s$-hedron (polar s-seit) of the $\left\{F_{i}\right\}$. It corresponds to a collection of hyperplanes in $\mathbb{P}^{n}$ which stands in some geometric relation to the system of hypersurfaces defined by the $F_{i}$. The precise nature of this relation is very sensitive to the values 
$(n, d, r, s)$, but in any event it is invariant under the automorphisms of $\mathbb{P}^{n}$.

For instance, in the example above, let $\Pi_{i}$ be the hyperplane defined by $L_{i}=0$. Then the $n+1$ points

$$
P_{k}=\Pi_{1} \cap \cdots \cap \widehat{\Pi}_{k} \cdots \cap \Pi_{n+1}, \quad(k=1, \ldots, n+1)
$$

are exactly the vertices of the singular quadrics belonging to the pencil $\left\{F_{1}+\lambda F_{2}=0\right\}_{\lambda \in \mathbb{P}^{1}}$.

1.1. A Summary of Results. Fix degree $d$ forms $\left\{F_{1}, \ldots, F_{r}\right\}$ as above. Then the polar $s$-hedra of this collection move in an algebraic family, denoted by $\mathcal{A}$. (See Definition 2.5 et seq. for the precise statement.) In this note we deduce results about the birational structure of $\mathcal{A}$ for several specific quadruples $(n, d, r, s)$, in each case assuming that the $F_{i}$ are chosen generally. A parameter count shows that the dimension of the variety $\mathcal{A}$ is 'expected' to be $s(n+r)-r\left(\begin{array}{c}n+d \\ d\end{array}\right)$ (more on this in 92 below). For the quadruples

$$
(2,4,2,8),(2,3,4,7),(3,2,6,7)(2,3,7,8),
$$

it is shown here that $\mathcal{A}$ is a rational variety of expected dimension. For the cases

$$
(2,3,8,8),(3,2,7,7),(2,4,3,9)(3,3,2,8),(2,3,3,6),
$$

the variety $\mathcal{A}$ is expected to be (and is) a finite set of points; in each case we determine its cardinality. The calculation for $(2,3,3,6)$ was done by Franz London over a century ago; a more rigorous and modern version of his proof is given here.

Along the way, we deduce some miscellaneous results for the quadruples

$$
(2,3,2,6),(2,4,2,8),(3,2,3,9) .
$$

For instance, the result for $(2,3,2,6)$ says the following: let $F_{1}, F_{2}$ be two general ternary cubics and $E$ a smooth planar cubic curve apolar to $F_{1}, F_{2}$ (in the sense explained below). Then $E$ passes through exactly three sextuples in $\mathcal{A}$.

In each of the cases above, there is a specific feature of the free resolution of $s$ general points in $\mathbb{P}^{n}$ which is exploited to deduce the answer. For the arguments to work smoothly, we require a technical condition on the polar $s$-hedra, namely that they be 'resolution-general' (in the sense of Definition 2.4). Although the specific technique used 
depends on the case at hand, two general themes are identifiable: the geometry of associated points and intersection theory on symmetric products of elliptic curves. I do not know of any technique which would apply uniformly to all $(n, d, r, s)$.

This subject is broadly referred to as 'reduction to canonical form' or 'Waring's problem for algebraic forms'; see [2, 6, 15, 20, for an introduction and further references. The paper [23] is an excellent compendium of known results about the structure of $\mathcal{A}$ when $r=1$. For a discussion of ternary cubics (the case $n=2, d=3$ ), see [22, 24].

AcKnowledgements: I would like to thank Anthony V. Geramita, Leslie Roberts and Queen's University for financial support while this work was in progress. The program Macaulay-2 has been useful, and I am grateful to its authors Dan Grayson and Mike Stillman.

\section{Preliminaries}

In this section we establish notation and describe the basic set-up of apolarity. The proofs may be found in [20, also see [7, 8, 15, 19].

The base field is $\mathbf{C}$. Let $V$ be an $(n+1)$-dimensional $\mathbf{C}$-vector space and consider the symmetric algebras

$$
R=\bigoplus_{i \geq 0} \operatorname{Sym}^{i} V^{*}, \quad S=\bigoplus_{j \geq 0} \operatorname{Sym}^{j} V .
$$

If $\underline{u}=\left\{u_{0}, \ldots, u_{n}\right\}, \quad \underline{x}=\left\{x_{0}, \ldots, x_{n}\right\}$, are dual bases of $V^{*}$ and $V$ respectively, then

$$
R=\mathbf{C}\left[u_{0}, \ldots, u_{n}\right], \quad S=\mathbf{C}\left[x_{0}, \ldots, x_{n}\right] .
$$

There are internal product maps $R_{i} \otimes S_{j} \stackrel{f_{i j}}{\longrightarrow} S_{j-i}$ (see e.g. [14, p. 476]), so $S$ acquires the structure of a graded $R$-module. With the identification $u_{\ell}=\frac{\partial}{\partial x_{\ell}}$, the internal product can be seen as partial differentiation: if $\varphi \in R_{i}$ and $F \in S_{j}$, then $f_{i j}(\varphi \otimes F)$ is obtained by applying the differential operator $\varphi\left(\frac{\partial}{\partial x_{0}}, \ldots, \frac{\partial}{\partial x_{n}}\right)$ to $F\left(x_{0}, \ldots, x_{n}\right)$. We will write $\varphi \circ F$ for $f_{i j}(\varphi \otimes F)$.

Let $\Lambda \subseteq S_{d}$ be an $r$-dimensional subspace of degree $d$ forms in the $\underline{x}$, defining a point in the Grassmannian $G\left(r, S_{d}\right)$. Let

$$
\Lambda^{\perp}=\{\varphi \in R: \varphi \circ F=0 \text { for every } F \text { in } \Lambda\} .
$$


Then $\Lambda^{\perp}=\bigoplus_{i} \Lambda_{i}^{\perp}$ is a graded ideal in $R$, with $\Lambda_{i}^{\perp}=R_{i}$ for $i>d$. (It follows that the quotient $R / \Lambda^{\perp}$ is an artin level algebra of socle degree $d$ and type $r$, but we will not use this explicitly.)

For $i \leq d$, the codimension of $\Lambda_{i}^{\perp}$ in $R_{i}$ equals the dimension of the image of the internal product map

$$
R_{d-i} \otimes \Lambda \longrightarrow S_{i}
$$

Hence

$$
\operatorname{dim} \Lambda_{i}^{\perp} \geq \max \left\{0, \operatorname{dim} R_{i}-r \cdot \operatorname{dim} R_{d-i}\right\} .
$$

Equality always holds for $i=d$, and it holds for all $i<d$ if $\Lambda$ is a general point in $G\left(r, S_{d}\right)$.

We will commonly use geometric language in the sequel, e.g., if $n=3$, then a point in $G\left(2, S_{4}\right)$ will be called a pencil of planar quartics.

Remark 2.1. If $\varphi \circ F=0$, then $\varphi, F$ were classically said to be apolar to each other; and sometimes the entire set-up is called apolarity. Of course, all of the above is subsumed in the statement that $R, S$ are dual Hopf algebras such that all structure maps are $S L(V)$-equivariant.

Henceforth we set $\mathbb{P}^{n}=\mathbb{P} S_{1}=$ Proj $R$. Usually $Z \subseteq \mathbb{P}^{n}$ will denote a closed subscheme with (saturated) ideal $I_{Z} \subseteq R$.

Definition 2.2. (cf. [20, Definition 5.1]) The scheme $Z$ is said to be apolar to $\Lambda$, if $I_{Z} \subseteq \Lambda^{\perp}$.

The point of the definition is the following:

Theorem 2.3 (Reye). If $Z$ consists of $s$ distinct points $\left\{L_{1}, \ldots, L_{s}\right\} \subseteq$ $\mathbb{P}^{n}$, then $Z$ is apolar to $\Lambda$ if and only if $\Lambda \subseteq \operatorname{span}\left\{L_{1}^{d}, \ldots, L_{s}^{d}\right\}$.

We would like to consider the family of such $Z$, but for technical reasons, we single out those schemes whose ideals are well-behaved.

Definition 2.4. A (zero-dimensional) length s scheme $Z \subseteq \mathbb{P}^{n}$ will be called resolution-general, if the graded Betti numbers in the minimal resolution of $I_{Z}$ are the same as those in the resolution of $s$ general points.

For instance, a length 7 subscheme $Z \subseteq \mathbb{P}^{2}$ is resolution-general iff its minimal resolution looks like

$$
0 \rightarrow R(-5) \oplus R(-4) \rightarrow R(-3)^{3} \rightarrow R \rightarrow R / I_{Z} \rightarrow 0 .
$$

In particular, $Z$ does not lie on a conic. 
Definition 2.5. A zero-dimensional scheme $Z \subseteq \mathbb{P}^{n}$ will be called a polar polyhedron of $\Lambda$, if it is apolar to $\Lambda$ and resolution-general.

Let $\operatorname{Hilb}\left(s, \mathbb{P}^{n}\right)$ be the Hilbert scheme parametrising length $s$ subschemes of $\mathbb{P}^{n}$. Let $\mathcal{A}(s, \Lambda)$ denote the set of polar $s$-hedra of $\Lambda$, it is then a constructible subset of $\operatorname{Hilb}\left(s, \mathbb{P}^{n}\right)$. We will write $\mathcal{A}$ for $\mathcal{A}(s, \Lambda)$ if no confusion is likely.

Remark 2.6. In the literature there is no unanimity on the definition of a 'polar polyhedron', in particular the approaches in [7] and [23] are different from ours and from each other. It is understood that if $Z=\left\{L_{1}, \ldots, L_{s}\right\}$ are $s$ general points, then morally $Z$ should count as a polar $s$-hedron of any $\Lambda \subseteq \operatorname{span}\left\{L_{i}^{d}\right\}$. However, it is not obvious which degenerations of $Z$ should be allowed, and it seems that (within reason) we should tailor our definition to the specific problem at hand. Many of our results depend on a free resolution of $I_{Z}$, and hence 'resolution-general' seems to be the most suitable notion. This issue never arises in [22], because there it is tacitly assumed that all geometric configurations are nondegenerate.

If $\mathcal{A}(s, \Lambda)$ is nonempty, so is $\mathcal{A}(t, \Lambda)$ for any $t>s$. It is the case that every $\Lambda$ in $G\left(r, S_{d}\right)$ admits a polar $\left(\begin{array}{c}n+d \\ d\end{array}\right)$-hedron. An elementary parameter count (see [2]) shows that a general $\Lambda$ in $G\left(r, S_{d}\right)$ will admit a polar $s$-hedron only if

$$
s \geq \frac{r\left(\begin{array}{c}
n+d \\
d
\end{array}\right)}{n+r} .
$$

Definition 2.7. A quadruple $(n, d, r, s)$ which satisfies (3) is said to be nondegenerate, if a general $\Lambda$ admits a polar $s$-hedron.

A quadruple satisfying (3) is degenerate if the set $\{\Lambda: \mathcal{A}(s, \Lambda) \neq \emptyset\}$ fails to be dense in $G\left(r, S_{d}\right)$. Very few such examples are known (see 2. for the list), but none of them is without its geometric peculiarity. In general it is not trivial to prove that a particular quadruple is nondegenerate.

For $r=1$, we have the following classification theorem by Alexander and Hirschowitz.

Theorem 2.8 (see [19]). Assuming $r=1$ and $d>2$, the only degenerate cases are $(n, d, s)=(2,4,5),(3,4,9),(4,4,14)$ and $(4,3,7)$.

For $r>1$ we have the following results by Dionisi and Fontanari. 
Theorem 2.9. Assume $r>1$. Then

(i) for $n=2$, the only degenerate quadruple is $(2,3,2,5)$;

(ii) there are no degenerate quadruples with $r \geq n+1$.

The proofs may be found in 4, 12] respectively. Part (i) was claimed by Terracini [27, but his proof is obscure.

If $(n, d, r, s)$ is nondegenerate, then with a slight abuse of notation we will write $\mathcal{A}$ for $\mathcal{A}(s, \Lambda)$, where $\Lambda$ is understood to be a general point of $G\left(r, S_{d}\right)$. It has dimension $s(n+r)-r\left(\begin{array}{c}n+d \\ d\end{array}\right)$.

\section{Associated systems of points}

Recall ([10, p. 313]) that if $\Gamma$ is a zero-dimensional Gorenstein scheme, then any closed subscheme $\Gamma^{\prime} \subseteq \Gamma$ has a residual scheme $\Gamma^{\prime \prime} \subseteq \Gamma$, such that

$$
\operatorname{deg} \Gamma^{\prime}+\operatorname{deg} \Gamma^{\prime \prime}=\operatorname{deg} \Gamma .
$$

In particular this applies if $\Gamma$ is a (global) complete intersection in $\mathbb{P}^{n}$, which is the only case we will need.

Now let $\Lambda$ denote a general pencil of planar quartics. Then $\mathcal{A}=$ $\mathcal{A}(8, \Lambda)$ is 2 -dimensional; we will show that it is rational. Every $Z \in \mathcal{A}$ has a Hilbert-Burch resolution

$$
0 \rightarrow R(-5)^{2} \stackrel{\mu}{\rightarrow} R(-4) \oplus R(-3)^{2} \rightarrow R \rightarrow R / I_{Z} \rightarrow 0 .
$$

(See 3] for the basic theory behind the Hilbert-Burch theorem.) In particular $\operatorname{dim}\left(I_{Z}\right)_{3}=2$, so $Z$ has an associated point $\alpha(Z)$, defined to be the residual intersection of cubics passing through $Z$. The matrix of the map $\mu$ has the form

$$
M=\left[\begin{array}{lll}
\underline{2} & \underline{2} & \underline{1} \\
\underline{2} & \underline{2} & \underline{1}
\end{array}\right],
$$

with the convention that $\underline{j}$ stands for a degree $j$ form.

Theorem 3.1. Let $\Lambda$ be a general pencil of planar quartics. Then the morphism $\alpha: \mathcal{A} \longrightarrow \mathbb{P}^{2}$ admits a rational inverse, hence $\mathcal{A}$ is a rational surface.

ProOF. Fix a general point in the image of $\alpha$, by change of coordinates we assume it to be $P=[0,0,1]$. We would like to show that there is a unique resolution general length 8 scheme $Z$ with associated point $P$. 
Now $P$ is defined by the vanishing of the rightmost column in (4), hence, after row-operations, $M$ can be brought into the form

$$
M=\left[\begin{array}{lll}
q_{1} & q_{2} & u_{0} \\
q_{3} & q_{4} & u_{1}
\end{array}\right], \quad q_{i} \in R_{2} .
$$

We start with the 24-dimensional vector space of $2 \times 2$ matrices

$$
V_{1}=\left\{N=\left[\begin{array}{ll}
q_{1} & q_{2} \\
q_{3} & q_{4}
\end{array}\right]: q_{i} \in R_{2}\right\}
$$

For $N \in V_{1}$, write

$$
\theta_{N}=u_{1} q_{1}-u_{0} q_{3}, \theta_{N}^{\prime}=u_{1} q_{2}-u_{0} q_{4}, \omega_{N}=q_{1} q_{4}-q_{2} q_{3}
$$

and let $J_{N}$ be the ideal generated by $\theta_{N}, \theta_{N}^{\prime}, \omega_{N}$. Thus $V_{1}$ is a parameter space for all Hilbert-Burch matrices as above. For a dense open set of elements $N$ in $V_{1}$, the ideal $J_{N}$ defines a planar length 8 scheme.

We let $G L_{2}(\mathbf{C})$ act on $V_{1}$ by right multiplication, i.e., for $g=$ $\left[\begin{array}{cc}\alpha & \beta \\ \gamma & \delta\end{array}\right] \in G L_{2}$, and $N$ as above,

$$
N g=\left[\begin{array}{ll}
q_{1} \alpha+q_{2} \gamma & q_{1} \beta+q_{2} \delta \\
q_{3} \alpha+q_{4} \gamma & q_{3} \beta+q_{4} \delta
\end{array}\right]
$$

Define $V_{2}=\left\{N \in V_{1}: \theta_{N}, \theta_{N}^{\prime} \in \Lambda_{3}^{\perp}\right\}$, which is a 12-dimensional subspace of $V_{1}$. (If $F \in \Lambda$, then $\theta_{N} \circ F=\theta_{N}^{\prime} \circ F=0$ is a set of six linear equations. In all, $V_{2}$ is defined by 12 linear equations which are independent for a general $\Lambda$, hence $\operatorname{dim} V_{2}=12$.) Inside $V_{2}$, there is a 6-dimensional subspace

$$
V_{3}=\left\{\left[\begin{array}{ll}
a u_{0} & b u_{0} \\
a u_{1} & b u_{1}
\end{array}\right]: a, b \in R_{1}\right\}
$$

(Since $\theta_{N}, \theta_{N}^{\prime}=0$ for $N \in V_{3}$, the containment $V_{3} \subseteq V_{2}$ is clear.) Form the 6 -dimensional space $W=V_{2} / V_{3}$. For $N \in V_{2}$, write $[N]$ for the corresponding point in the projective space $\mathbb{P} W \simeq \mathbb{P}^{5}$. Since $V_{3} \subseteq V_{2} \subseteq V_{1}$ are inclusions of $G L_{2}$-modules, $W$ is also a (right) $G L_{2^{-}}$ module; in particular $P G L_{2}$ acts on $\mathbb{P} W$. The point of this construction lies in the following lemma:

Lemma 3.2. $\quad$ (i) If $N, \tilde{N} \in V_{2}$ are such that $[N],[\tilde{N}]$ lie in the same $P G L_{2}$-orbit of $\mathbb{P} W$, then $J_{N}=J_{\widetilde{N}}$. 
(ii) Let $Z \in \alpha^{-1}(P)$. Consider two minimal resolutions of $I_{Z}$ with corresponding Hilbert-Burch matrices $M, \widetilde{M}$, and let $N, \widetilde{N}$ denote their leftmost minors. Then $[N],[\tilde{N}]$ lie in the same $P G L_{2^{-}}$ orbit in $\mathbb{P} W$.

Proof. By straightforward calculation,

$$
\theta_{N g}=\alpha \theta_{N}+\gamma \theta_{N}^{\prime}, \quad \theta_{N g}^{\prime}=\beta \theta_{N}+\delta \theta_{N}^{\prime}, \quad \omega_{N g}=\operatorname{det}(g) \omega_{N},
$$

$$
\begin{aligned}
& \text { so } J_{N}=J_{N g} \text {. Let } Q=\left[\begin{array}{lll}
a & u_{0} & b u_{0} \\
a & u_{1} & b u_{1}
\end{array}\right] \in V_{3} \text {. Then } \\
& \theta_{N+Q}=\theta_{N}, \quad \theta_{N+Q}^{\prime}=\theta_{N}^{\prime}, \quad \omega_{N+Q}=\omega_{N}-a \theta_{N}^{\prime}+b \theta_{N},
\end{aligned}
$$

so $J_{N+Q}=J_{N}$. This proves (i).

Any two minimal resolutions of $I_{Z}$ are isomorphic (see [9, §20.1]), which translates into the statement that $N$ and some $G L_{2}$-translate of $\widetilde{N}$ must differ by an element of $V_{3}$. This says that $[N],[\widetilde{N}]$ must be in the same orbit, which is (ii).

Now define a subvariety $Y=\left\{[N] \in \mathbb{P} W: \omega_{N} \circ \Lambda=0\right\} \subseteq \mathbb{P} W$. Formulae (8) imply that $\omega_{N+Q} \circ \Lambda=0 \Longleftrightarrow \omega_{N} \circ \Lambda=0$ (since $\theta_{N} \circ \Lambda=\theta_{N}^{\prime} \circ \Lambda=0$ ), hence this definition is meaningful. The inclusion $Y \subseteq \mathbb{P} W$ is a $P G L_{2}$-stable by formulae (7). By the previous lemma, each $Z \in \alpha^{-1}(P)$ defines an orbit $\Omega_{Z} \subseteq Y$. The $P G L_{2}$-stabilizer of a point in $\Omega_{Z}$ is trivial, hence $\operatorname{dim} \Omega_{Z}=3$. The union of $\left\{\Omega_{Z}\right\}_{Z \in \alpha^{-1}(P)}$ fills a dense open subset in $Y$. Hence it is enough to show that $Y$ contains only one three-dimensional component, this will imply that $\alpha^{-1}(P)$ is singleton. Define

$$
\begin{aligned}
& \Gamma_{1}=\left\{[N] \in \mathbb{P} W: N=\left[\begin{array}{ll}
q_{1} & 0 \\
q_{3} & 0
\end{array}\right] \text { for some } q_{i} \text { and } \theta_{N} \circ \Lambda=0\right\}, \\
& \Gamma_{2}=\left\{[N] \in \mathbb{P} W: N=\left[\begin{array}{ll}
0 & q_{2} \\
0 & q_{4}
\end{array}\right] \text { for some } q_{i} \text { and } \theta_{N}^{\prime} \circ \Lambda=0\right\},
\end{aligned}
$$

each of which is a copy of $\mathbb{P}^{2}$ in $Y$. Define a birational map $h: \Gamma_{1} \longrightarrow$ $\Gamma_{2}$ as follows. Let $[N] \in \Gamma_{1}$, then there is a 4-dimensional family of solutions $\left(q_{2}, q_{4}\right)$ to the equations

$$
\theta_{N}^{\prime} \circ \Lambda=\omega_{N} \circ \Lambda=0 .
$$

(This is so because $q_{2}, q_{4}$ together depend upon 12 parameters and there are 8 equations.) However, if $\left(q_{2}, q_{4}\right)$ is one such solution, then $\left(q_{2}+a u_{0}, q_{4}+a u_{1}\right)$ is also one for any $a \in R_{1}$, and this accounts for 
all the solutions. Hence the class in $\mathbb{P} W$ of the matrix $\left[\begin{array}{ll}0 & q_{2} \\ 0 & q_{4}\end{array}\right]$ is uniquely determined. We define $h([N])$ to be this class. (The reader should verify that this definition is independent of the choice of coset representative for $[N]$.) Now a general element in $Y$ can be written as a sum $[N]+[h(N)]$ for $[N] \in \Gamma_{1}$, i.e., the ruled join of $\Gamma_{1}, \Gamma_{2}$ along $h$ contains a dense open subset of $Y$. Since this join is irreducible (it is the image of the Segre imbedding $\mathbb{P}^{2} \times \mathbb{P}^{1} \subseteq \mathbb{P}^{5}$ ), we are done.

The argument for the following proposition is similar. As before, $(2,3,4,7)$ is nondegenerate by Theorem 2.9 .

Proposition 3.3. Let $\Lambda$ be a general web of planar cubics. Then $\mathcal{A}(7, \Lambda)$ is a rational surface.

Proof. The Hilbert-Burch matrix for $Z \in \mathcal{A}$ is $\left[\begin{array}{lll}\underline{1} & \underline{1} & \underline{1} \\ \underline{2} & \underline{2} & \underline{2}\end{array}\right]$. For a general $Z$, the linear forms in the top row are independent, hence after column operations we can assume the matrix to be

$$
\left[\begin{array}{ccc}
u_{0} & u_{1} & u_{2} \\
q_{0} & q_{1} & q_{2}
\end{array}\right], \quad q_{i} \in R_{2} .
$$

Let $V_{1}$ denote the 18-dimensional vector space $\left\{\left[q_{0}, q_{1}, q_{2}\right]: q_{i} \in R_{2}\right\}$, and $V_{2}$ the 3 -dimensional subspace $\left\{\left[a u_{0}, a u_{1}, a u_{2}\right]: a \in R_{1}\right\}$. Let $W=V_{1} / V_{2}$. Then the 12 equations $\left\{\left(u_{i} q_{j}-u_{j} q_{i}\right) \circ \Lambda=0\right\}$ cut out a 2-plane in $\mathbb{P} W$ which is birational to $\mathcal{A}$.

Now let $(n, d, r, s)=(3,2,6,7)$, we will show that $\mathcal{A}$ is birational to the projective 3 -space. The ideal of every $Z \in \mathcal{A}$ is generated by three quadrics and and a cubic. The associated point $\alpha(Z)$ is defined to the residual intersection of the quadrics through $Z$.

Proposition 3.4. Let $\Lambda$ be a general point of $G\left(6, S_{2}\right)$. Then the map $\alpha: \mathcal{A} \longrightarrow \mathbb{P}^{3}$ is birational.

Proof. Let $Z$ be a resolution-general scheme of length 7 . It is apolar to $\Lambda$ iff the three generating quadrics lie in $\Lambda_{2}^{\perp}$.

Let $P$ be a general point of $\mathbb{P}^{3}$, and let $W \subseteq \Lambda_{2}^{\perp}$ be the 3-dimensional subspace of forms vanishing at $P$. Then $W$ defines a length 8 scheme $Y$. Now the residual scheme of $P$ in $Y$ is the only point of $\mathcal{A}$ mapping to $P$. 
Remark 3.5. The case $(2,3,7,8)$ has a similar geometry, where $\mathcal{A}$ is birational to $\mathbb{P}^{2}$. For $(2,3,8,8)$ (resp. $\left.(3,2,7,7)\right), \mathcal{A}$ is a finite set consisting of 9 (resp. 8) points.

\section{Symmetric Products of Elliptic Curves}

For the examples in this section, the determination of $\mathcal{A}$ reduces to an intersection-theoretic calculation on the symmetric product of an elliptic curve. If $E$ is a smooth projective curve, then $E^{(m)}$ will denote its $m$-th symmetric product. This is a smooth projective variety whose points are naturally seen as effective degree $m$ divisors on $E$.

Let $\Lambda$ be a general net of planar quartics. Since $(2,4,3,9)$ is nondegenerate, $\mathcal{A}$ is a finite set. In the next theorem we calculate its cardinality.

Theorem 4.1. Let $\Lambda$ be a general net of planar quartics. Then $\Lambda$ admits 4 polar enneahedra.

Proof. The ideal of $Z \in \mathcal{A}$ is generated by one cubic and 3 quartics. The space $\Lambda_{3}^{\perp}$ is one-dimensional, i.e., $\Lambda$ is apolar to a unique cubic curve $E \subseteq \mathbb{P}^{2}$. Since $\Lambda$ is general, we may (and will) assume that $E$ is smooth. If $H$ denotes the hyperplane divisor on $E$, then we have an identification $H^{0}(E, 4 H)=R_{4} /\left(I_{E}\right)_{4}$. This is a 12-dimensional space, denoted $U$.

Let $W=\Lambda_{4}^{\perp} /\left(I_{E}\right)_{4}$, which is a 9-dimensional space inside $U$. Every scheme $Z \subseteq \mathbb{P}^{2}$ of length 9 which is apolar to $\Lambda$ is contained in $E$, and thus defines an effective divisor on $E$. Then the 3 -dimensional space $H^{0}(E, 4 H-Z)$, which is a priori inside $U$, is in fact contained in $W$. The argument shows that the following diagram is a fibre square:

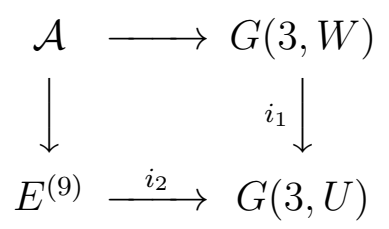

Here $i_{1}$ is the natural inclusion and $i_{2}(Z)=H^{0}(E, 4 H-Z)$. Since the images of both inclusions have complementary codimensions, it is enough to take the intersection of their classes inside $H^{*}(G(3, U), \mathbf{Z})$ in order to calculate the degree of $\mathcal{A}$ as a zero-cycle.

Conventions. The notation for Schubert calculus follows [13, §14.7]. We refer to [1] for some basic cohomological calculations on curves. 
If $X_{1}, X_{2}$ are varieties, then denote projections by $\pi_{i}: X_{1} \times X_{2} \longrightarrow$ $X_{i}$. All cohomology is with $\mathbf{Z}$-coefficients. If $\alpha$ is a class in $H^{*}\left(X_{1}\right)$ (resp. $H^{*}\left(X_{2}\right)$ ), then its pullback to $H^{*}\left(X_{1} \times X_{2}\right)$ is denoted $\alpha \otimes 1$ (resp. $1 \otimes \alpha$ ). Cup product is written as juxtaposition.

Firstly, we should find the rank 3 subbundle of $U \otimes \mathcal{O}_{E^{(9)}}$ which defines the inclusion $i_{2}$. Let $\Delta$ denote the universal divisor on $E^{(9)} \times E$ (see [1, Ch. IV]), so that $\left.\Delta\right|_{\{Z\} \times E}=Z \times E$. Define a line bundle $\mathcal{M}=\pi_{2}^{*}\left(\mathcal{O}_{E}(4 H)\right) \otimes \mathcal{O}(-\Delta)$ on $E^{(9)} \times E$. Applying $\pi_{1 *}$ to the inclusion

$$
\mathcal{M} \subseteq \pi_{2}^{*}\left(\mathcal{O}_{E}(4 H)\right)
$$

we have

$$
(\mathcal{G}=) \pi_{1 *}(\mathcal{M}) \subseteq U \otimes \mathcal{O}_{E^{(9)}}
$$

A moment's reflection will show that $i_{2}$ is induced by the last inclusion.

The image of $i_{2}$ has class $\{3,3,3\}$. Hence by the Jacobi-Trudi identity, the class of $\mathcal{A}$ in $H^{18}\left(E^{(9)}\right)$ is given by $c_{3}\left(\mathcal{G}^{*}\right)^{3}$, which we now calculate.

The cohomology rings of $E$ and $E^{(9)}$. Let $\delta_{1}, \delta_{2} \in H^{1}(E)$ be a symplectic basis, it will then generate $H^{*}(E)$. The product $\eta=\delta_{1} \delta_{2} \in$ $H^{2}(E)$ is the class of a point.

Let $\mathcal{L}$ be a Poincaré line bundle ([1, Ch. IV]) on $E \times \operatorname{Pic}^{9}(E)$, then $\mathcal{E}=\pi_{2 *}(\mathcal{L})$ is a rank 9 bundle on $\operatorname{Pic}^{9}(E)$. Fix an isomorphism $\operatorname{Pic}^{9}(E)=E$, then by the calculation of [1, p. 336], $c_{1}(\mathcal{E})=-\eta$. Now let $\xi=c_{1}\left(\mathcal{O}_{\mathbb{P} \mathcal{E}}(1)\right) \in H^{2}(\mathbb{P} \mathcal{E})$. With the identification $\mathbb{P} \mathcal{E}=E^{(9)}$, the ring $H^{*}\left(E^{(9)}\right)$ is generated by $\xi$ and (the pullbacks of) $\delta_{1}, \delta_{2}$, subject to the relation $\xi^{9}=\xi^{8} \eta$.

The Chern class of $\mathcal{M}$ and G-R-R. Let

$$
-\gamma=\left(\delta_{1} \otimes 1\right)\left(1 \otimes \delta_{2}\right)-\left(\delta_{2} \otimes 1\right)\left(1 \otimes \delta_{1}\right)
$$

a class in $H^{1,1}\left(E^{(9)} \times E\right)$. By [1, p. 337-338],

$$
c_{1}(\mathcal{O}(\Delta))=\xi \otimes 1+\gamma+9(1 \otimes \eta)
$$

hence

$$
c_{1}(\mathcal{M})=-\xi \otimes 1-\gamma+3(1 \otimes \eta) .
$$

Now we apply Grothendieck-Riemann-Roch to $\mathcal{M}$ along the projection $E^{(9)} \times E \stackrel{\pi_{1}}{\longrightarrow} E^{(9)}$. Thus

$$
\operatorname{ch}\left(\pi_{1 !} \mathcal{M}\right) \operatorname{td}\left(E^{(9)}\right)=\pi_{1 *}\left(\operatorname{ch}(\mathcal{M}) \operatorname{td}\left(E^{(9)} \times E\right)\right) .
$$


Since $R^{i} \pi_{1 *} \mathcal{M}=0$ for $i>0$ and $\operatorname{td}(E)=1$, this simplifies to

$$
\operatorname{ch}(\mathcal{G})=\pi_{1 *}\left(e^{c_{1}(\mathcal{M})}\right) .
$$

Let $n_{i}$ denote the $i$-th Newton class of $\mathcal{G}$ (i.e., the sum of $i$-th powers of the Chern roots of $\mathcal{G})$, then $\operatorname{ch}(\mathcal{G})=\sum_{i \geq 0} n_{i} / i$ !. Now we expand the exponential series, and apply $\pi_{1 *}$ term by term, to get

$$
\begin{aligned}
n_{0}=3, \quad n_{1} & =\frac{1}{2}(-6 \xi-2 \eta), \\
n_{2}=\frac{1}{3}\left(9 \xi^{2}+6 \xi \eta\right), \quad n_{3} & =\frac{1}{4}\left(-12 \xi^{3}-12 \xi^{2} \eta\right) .
\end{aligned}
$$

Then

$$
c_{3}(\mathcal{G})=\frac{1}{6} n_{1}^{3}-\frac{1}{2} n_{1} n_{2}+\frac{1}{3} n_{3}=-\left(\xi^{3}+\xi^{2} \eta\right) .
$$

Hence finally

$$
c_{3}\left(\mathcal{G}^{*}\right)^{3}=\left(\xi^{3}+\xi^{2} \eta\right)^{3}=4 \xi^{8} \eta .
$$

Since $\xi^{8} \eta$ is the class of a point on $E^{(9)}$, we deduce that $\mathcal{A}$ has degree 4.

In order to show that $\mathcal{A}$ is reduced and hence consists of 4 geometric points, we use Kleiman's transversality result (see [18, Theorem 10.8]). We can reformulate the entire construction in the following way: start with a smooth $E$ and hence $U$, then specifying a codimension 3 subspace $W \subseteq U$ is tantamount to specifying $\Lambda$. Since $G(3, U)$ is a homogeneous space for $G L(U)$, the intersection is transversal for a general $W$, so $\mathcal{A}$ is reduced.

The next example is that of a pencil of cubic surfaces. We need to show that $(3,3,2,8)$ is nondegenerate, the proof is given in $\$ 6$.

Proposition 4.2. Let $\Lambda$ be a general pencil of cubic surfaces. Then $\Lambda$ admits 3 polar octahedra.

Proof. The calculation is very similar to Theorem 4.1. The ideal of 8 general points in $\mathbb{P}^{3}$ is generated by 2 quadrics and 4 cubics. Now $\Lambda_{2}^{\perp}$ is 2-dimensional, hence generates the ideal of a smooth normal elliptic quartic $E \subseteq \mathbb{P}^{3}$ apolar to $\Lambda$, and every $Z \in \mathcal{A}$ is in fact contained in E. Let

$$
U=R_{3} /\left(I_{E}\right)_{3}, \quad W=\Lambda_{3}^{\perp} /\left(I_{E}\right)_{3}
$$


which are spaces of dimension 12,10 respectively. Define $i_{1}, i_{2}$ as before, then the following diagram is a fibre square

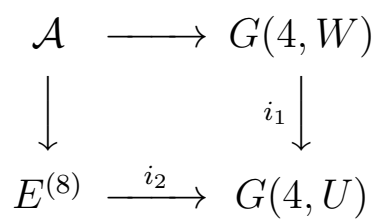

Now $i_{2}$ is induced by a rank 4 bundle $\mathcal{G}$ on $E^{(8)}$. The class of $\mathcal{A}$ in $E^{(8)}$ equals

$$
c_{4}\left(\mathcal{G}^{*}\right)^{2}=\left(\xi^{4}+\xi^{3} \eta\right)^{2}=3 \xi^{7} \eta .
$$

The argument for transversality is the same as before.

Using similar calculations, we can give alternate proofs of the following results by Schlesinger [26, p. 212]). The original argument uses $\vartheta$-functions.

Proposition 4.3 (Schlesinger). $\quad$ (1) Let $\Lambda$ be a general pencil of planar cubics. Fix a general elliptic curve $E \subseteq \mathbb{P}^{2}$ apolar to $\Lambda$. Then there are 3 polar hexahedra of $\Lambda$ which are contained in E.

(2) Let $\Lambda$ be a general pencil of planar quartics. Fix a general elliptic curve $E \subseteq \mathbb{P}^{2}$ apolar to $\Lambda$. Then there are 3 polar octahedra of $\Lambda$ which are contained in $E$.

Proof. We will only prove (1), the argument for (2) is identical in essence. Recall that the ideal of 6 general planar points is generated by 4 cubics. Since $(2,3,2,6)$ is nondegenerate ${ }^{1}, \mathcal{A}(6, \Lambda)$ is 4 -dimensional. Consider the incidence correspondence

$$
\Phi \subseteq \mathcal{A} \times \mathbb{P} \Lambda_{3}^{\perp}, \quad \Phi=\{(Z, E): Z \subseteq E\} .
$$

The projection $\pi_{1}: \Phi \longrightarrow \mathcal{A}$ is generically a $\mathbb{P}^{3}$-bundle, so $\operatorname{dim} \Phi=7$. Fix a general elliptic curve $E$ apolar to $\Lambda$, and consider the diagram

$$
\begin{gathered}
G\left(3, \Lambda_{3}^{\perp} /\left(I_{E}\right)_{3}\right) \\
i_{1} \downarrow \\
E^{(6)} \stackrel{i_{2}}{\longrightarrow} G\left(3, R_{3} /\left(I_{E}\right)_{3}\right)
\end{gathered}
$$

As usual, $i_{1}$ is the inclusion and $i_{2}(Z)=H^{0}(E, 3 H-Z)$. Then $i_{2}(Z)$ lies in the image of $i_{1}$, iff $Z$ is apolar to $\Lambda$. Calculating as before, the product [image $i_{1}$ ]. [image $i_{2}$ ] equals thrice the class of a point. Hence

\footnotetext{
${ }^{1}$ This is the smallest $s$ possible, because $(2,3,2,5)$ is degenerate by [2].
} 
$\pi_{2}^{-1}(E)$ must be nonempty. This implies that $\pi_{2}: \Phi \longrightarrow \mathbb{P} \Lambda_{3}^{\perp}\left(\simeq \mathbb{P}^{7}\right)$ is dominant. But then it is generically finite, hence for a general $E$, the fibre $\pi_{2}^{-1}(E)$ consists of 3 points.

It is shown in [2] (using a machine calculation) that $(5,2,3,9)$ is nondegenerate. Now there is a (unique) elliptic sextic curve passing through 9 general points of $\mathbb{P}^{5}$. (The classical reference is [25], also see [5] for a proof using Gale duality.) Hence if $\Lambda$ is a general net of quadrics in $\mathbb{P}^{5}$ and $Z$ a set of 9 general points apolar to $\Lambda$, then the elliptic sextic passing through $Z$ is apolar to $\Lambda$.

Proposition 4.4. Let $\Lambda$ be a general net of quadrics in $\mathbb{P}^{5}$. Fix a general elliptic sextic curve $E \subseteq \mathbb{P}^{5}$ apolar to $\Lambda$. Then there are 4 polar enneahedra of $\Lambda$ which are contained in $E$.

Proof. Similar to above. Use the fact that the ideal of 9 general points (resp. an elliptic sextic curve) is generated by 12 (resp. 9) quadrics.

\section{The $(2,3,3,6)$ CASE}

Now we come to London's beautiful calculation in [22], where he determines the number of polar hexahedra of a general net of cubic curves. I have rewritten the proof so as to make it more transparent, but all the key ideas are already in the original.

Let $\Lambda$ be such a net. By Theorem 2.9(i), $\Lambda$ has a finite number of polar hexahedra. We will count them by setting up a correspondence on a certain elliptic curve.

5.1. We begin by motivating the constructions which are to follow. Say $\left\{F_{1}, F_{2}, F_{3}\right\}$ is a basis of $\Lambda$ and $Z=\left\{L_{1}, \ldots, L_{6}\right\}$ one of its polar hexahedra. We have expressions

$$
F_{j}=c_{1 j} L_{1}^{3}+\cdots+c_{6 j} L_{6}^{3}, \quad j=1,2,3 .
$$

Let $\psi \in R_{2}$ be the form which defines the conic passing through $\left\{L_{2}, \ldots, L_{6}\right\} \subseteq \mathbb{P} S_{1}$. Since $\psi$ annihilates $L_{2}^{3}, \ldots, L_{6}^{3}$, we have $\psi \circ F_{j}=$ constant $\times L_{1}$ for every $j$, so $\psi \circ \Lambda$ is only a 1-dimensional vector space. It will be seen below $(\$ 5.2)$ that all $\psi$ with this property lie on a curve. Similarly if $l_{1}, l_{1}^{\prime} \in R_{1}$ annihilate $L_{1}$, then the six derivatives $\left\{l_{1} \circ F_{j}, l_{1}^{\prime} \circ F_{j}: j=1,2,3\right\}$ span only a 5 -dimensional space. It will be seen below (\$5.3) that all 2-dimensional spaces $\operatorname{span}\left\{l_{1}, l_{1}^{\prime}\right\} \subseteq R_{1}$ with this property lie on a curve, isomorphic to the previous one. 
5.2. Now we come to the actual constructions. The symbol $(\leftrightarrow)$ will appear frequently, it is explained in Remark 5.2. Consider the vector bundle morphism on $\mathbb{P} R_{2}\left(=\mathbb{P}^{5}\right)$

$$
f_{23}: \mathcal{O}_{\mathbb{P}^{5}}(-1) \otimes \Lambda \longrightarrow S_{1}
$$

coming from the internal product map of \$2, Define the degeneracy locus $\Psi=\left\{\operatorname{rank} f_{23} \leq 1\right\}$. For a general $\Lambda$, it is a degree 6 normal elliptic curve in $\mathbb{P}^{5}(\leftrightarrow)$. Note that $\Lambda_{2}^{\perp}=0$ by the generality of $\Lambda$, so rank $f_{23}$ is exactly 1 at each $\psi \in \Psi$.

5.3. Now identify $\mathbb{P} S_{1}$ with the Grassmannian $G\left(2, R_{1}\right)$, the latter is equipped with a rank two tautological bundle $\mathcal{B} \subseteq R_{1} \otimes \mathcal{O}_{G}$. The internal product $f_{13}$ gives a morphism

$$
f_{13}: \mathcal{B} \otimes \Lambda \longrightarrow S_{2}
$$

The locus $E=\left\{\operatorname{rank} f_{13} \leq 5\right\}=\left\{\operatorname{det} f_{13}=0\right\}$ is given by a section of $\mathcal{O}_{\mathbb{P} S_{1}}(3)$, hence it is a smooth $(\leftarrow)$ degree 3 curve in $\mathbb{P} S_{1}$. By the generality of $\Lambda$, the rank of $f_{13}$ is exactly 5 at every $L \in E(\leftrightarrow)$.

5.4. We have an isomorphism

$$
\alpha: E \longrightarrow \Psi
$$

defined as follows: let $L \in E$, and $U=L^{\perp}$. By hypothesis, the space $f_{13}(U \otimes \Lambda)$ is 5 -dimensional, so it is annihilated by a unique form in $\mathbb{P} R_{2}$, we declare $\alpha(L)$ to be this form. It is clear that $f_{23}(\alpha(L) \otimes \Lambda)$ is only 1-dimensional (since $U$ annihilates it), so $\alpha(L) \in \Psi$.

If $Z$ is as in $\$ 5.1$ above, then $\alpha\left(L_{1}\right)$ is the conic envelope containing the lines defined by $L_{2}, \ldots, L_{6}$.

5.5. Define a correspondence $\mathbb{T}$ on $E$ as follows: $(L, M) \in \mathbb{T}$ iff $M$ lies on the conic defined by $\alpha(L)$. For a fixed $L$, there are 6 positions of $M$ such that $(L, M) \in \mathbb{T}$. For a fixed $M$, the elements of $\Psi$ which vanish at $M$ lie on a hyperplane section of $\Psi$. Via $\alpha^{-1}$, the points of this hyperplane section correspond to 6 positions of $L$. This shows that $\mathbb{T}$ has degree $(6,6)$ and valence zero.

5.6. By the general theory of correspondences (see [16, §2.5]), there are 12 elements in $\mathbb{T}$ of the form $(L, L)$, they are called the united points of $\mathbb{T}$. Moreover $\mathbb{T}, \mathbb{T}^{-1}$ have 72 common points, i.e., pairs $(L, M)$ such that $(L, M),(M, L) \in \mathbb{T}$. Hence there are $72-12=60$ such pairs where $(L, M)$ are distinct. 
It is clear that starting from $Z$, the pairs $\left(L_{1}, L_{2}\right)$ etc. are common to $\mathbb{T}, \mathbb{T}^{-1}$. The next lemma says that the implication is reversible.

Lemma 5.1. Assume $(L, M),(M, L) \in \mathbb{T}$, and $L \neq M$. Let the conics $\alpha(L), \alpha(M)$ intersect in $\left\{P_{1}, P_{2}, P_{3}, P_{4}\right\}$. Then $Z=\left\{L, M, P_{1}, \ldots, P_{4}\right\}$ is a polar hexahedron of $\Lambda$.

ProOF. Recall that the ideal of 6 general points is generated by 4 cubics. Let $l, l^{\prime} \in R_{1}$ be generators of $L^{\perp}$, and $m, m^{\prime}$ of $M^{\perp}$. Consider the four cubic forms

$$
\left\{l \alpha(L), l^{\prime} \alpha(L), m \alpha(M), m^{\prime} \alpha(M)\right\} .
$$

They are linearly independent and each of them vanishes at all points of $Z$. Hence together they generate $\left(I_{Z}\right)_{3}$. Moreover, the definition of $\alpha$ implies that each of them annihilates $\Lambda$. Hence $I_{Z} \subseteq \Lambda^{\perp}$.

Now a polar hexahedron of $\Lambda$ gives $2\left(\begin{array}{c}6 \\ 2\end{array}\right)=30$ pairs $\left(L_{i}, L_{j}\right)$ common to $\mathbb{T}, \mathbb{T}^{-1}$. Alternately, starting from a common point we can reconstruct a polar hexahedron as shown above. Hence, following London, we conclude that $\Lambda$ has $60 \div 30=2$ polar hexahedra.

Remark 5.2. At several points in the proof we need to argue that our construction satisfies a certain 'good' property, for instance $\Psi$ has codimension 4 as expected and is smooth. This follows from the generality of $\Lambda$, as soon as we verify that it holds for a specific $\Lambda$. Such points are marked by $(\leftrightarrow)$, and I have verified the property in question by a direct computer calculation for a general net in the span of

$$
x_{0}^{3}, x_{1}^{3}, x_{2}^{3},\left(x_{0}+x_{1}+x_{2}\right)^{3},\left(x_{0}-x_{1}+x_{2}\right)^{3},\left(x_{0}-2 x_{1}+3 x_{2}\right)^{3} .
$$

This was carried out in Macaulay-2. For instance, to verify the last point in $\$ 5.3$, we choose two basis elements with indeterminate entries for an element of $G\left(2, R_{1}\right)$, represent $f_{13}$ by a matrix and check that the ideal defined by all $5 \times 5$ minors defines the empty scheme.

\section{Nondegeneracy of $(3,3,2,8)$}

To prove this result, we will use the notion of a grove, which was introduced in [2]. The general definition is meaningful for any $(n, d, r, s)$, but we will formulate it only for the case at hand.

Let $\underline{p}=\left\{p_{1}, \ldots, p_{8}\right\}$ (resp. $\left.\underline{Q}=\left\{Q_{1}, \ldots, Q_{8}\right\}\right)$ be points in $\mathbb{P}^{1}$ (resp. in $\mathbb{P}^{3}$ ). 
Definition 6.1. A grove for the data $p, Q$ is a linear system $\Gamma \subseteq$ $\mathbb{P} H^{0}\left(\mathbb{P}^{3}, \mathcal{O}_{\mathbb{P}}(3)\right)$ of projective dimension (say) $t$, satisfying the following conditions:

(1) The base locus of $\Gamma$ contains all the $Q_{i}$,

(2) $t=0$ or 1 , and

(3) either $t=0$ and the generator of $\Gamma$ is singular at all $Q_{i}$, or $t=1$ and there is an isomorphism $\gamma: \mathbb{P}^{1} \longrightarrow \Gamma$ such that for every $i$, the hypersurface $\gamma\left(p_{i}\right)$ is singular at $Q_{i}$.

Now [2, Theorem 2.6] says the following: the quadruple $(3,3,2,8)$ is nondegenerate iff there does not exist a grove for general points $\underline{p}, \underline{Q}$ as above. Existence of a grove is an open property of $\underline{p}, \underline{Q}$ (loc. cit.), so it is enough to exhibit some collection of points which does not admit a grove. I concede that the definition of a grove is awkward, in defence one can only say that it is a proof-generated concept in the sense of Lakatos (see [21, Appendix 2]). We begin with a preliminary lemma.

Lemma 6.2. Let $E$ be an elliptic curve and $\mathcal{M}$ a line bundle on $E$ of degree 4. Let $Q_{1}, \ldots, Q_{8}$ be distinct points on $E$. Then it is possible to find points $p_{1}, \ldots, p_{8}$ on $\mathbb{P}^{1}$, such that there is no morphism $f: E \longrightarrow$ $\mathbb{P}^{1}$ satisfying the following conditions:

A. $2 \leq \operatorname{deg} f \leq 4$, and if $\operatorname{deg} f=4$ then $f^{*}\left(\mathcal{O}_{\mathbb{P}^{1}}(1)\right) \simeq \mathcal{M}$;

B. the equality $f\left(Q_{i}\right)=p_{i}$ holds for at least $4+\operatorname{deg} f$ values of $i$.

Proof. Since $h^{0}(\mathcal{M})=4$, there are $\infty^{4} g_{4}^{1}$ 's coming from $\mathcal{M}$. However, modulo automorphisms of $\mathbb{P}^{1}$ there are $\infty^{5}$ octuples $\left(p_{1}, \ldots, p_{8}\right)$. Hence for a general octuple, there is no such map of degree 4 .

Similarly there are $\infty^{3}$ (resp. $\left.\infty^{1}\right) g_{3}^{1}$ 's (resp. $g_{2}^{1}$ 's) on E. Since (B) imposes 4 (resp. 3) conditions in these cases, for a general choice of $p_{i}$ none of the possibilities can hold. The lemma is proved.

Now $w, x, y, z$ be the coordinates in $\mathbb{P}^{3}$. Consider the normal elliptic quartic $E \subseteq \mathbb{P}^{9}$ defined by the two quadrics

$$
G_{1}=w x+x y+y z+z w, \quad G_{2}=w y+x z .
$$

Choose points

$$
\begin{aligned}
& Q_{1}=[1,0,0,0], \quad Q_{3}=[0,0,1,0], \quad Q_{5}=[-1,1,1,1], \quad Q_{7}=[1,1,-1,1], \\
& Q_{2}=[0,1,0,0], \quad Q_{4}=[0,0,0,1], \quad Q_{6}=[1,-1,1,1], \quad Q_{8}=[1,1,1,-1] \text {, }
\end{aligned}
$$

all lying on $E$, and the $p_{i}=\left[p_{i 1}, p_{i 2}\right]$ general in $\mathbb{P}^{1}$. 
Assume by way of contradiction that $\Gamma$ is a grove for the data. If $t=0$, then the generator of $\Gamma$ contains at least 16 points of $E$ (counting each $Q_{i}$ as two), hence it contains $E$ by Bézout's theorem.

Case 1. Assume that $\Gamma$ contains $E$ as a fixed component (with $t$ possibly 0 or 1 ). Then $\Gamma$ is spanned by two cubics of the form

$$
C_{1}=L_{1} G_{1}+L_{2} G_{2}, \quad C_{2}=L_{1}^{\prime} G_{1}+L_{2}^{\prime} G_{2}
$$

where $L_{1}, L_{1}^{\prime}$ etc are linear forms and $p_{i 1} C_{1}+p_{i 2} C_{2}$ is singular at $Q_{i}$ for $i=1, \ldots, 8$. (The case $C_{1}=$ (constant). $C_{2}$ corresponds to $t=0$.) An elementary linear algebra computation on the Jacobian matrix shows that this is impossible for general $p_{i}$.

Case 2. Assume that $E$ is not contained in the base locus of $\Gamma$ (hence necessarily $t=1)$. Let $\lambda$ be the linear series obtained by restricting $\Gamma$ to $E$ and removing the base divisor $\sum Q_{i}$. Thus $\lambda$ is a $g_{4}^{1}$. Let $f: E \longrightarrow \mathbb{P}^{1}$ be the corresponding morphism (of course, only welldefined up to automorphisms of $\left.\mathbb{P}^{1}\right)$. Let $H$ denote the hyperplane divisor on $E$ and $\mathcal{M}=\mathcal{O}_{E}\left(4 H-\sum Q_{i}\right)$.

Case 2.1. If $\lambda$ is base point free (i.e., if $\Gamma$ has no additional base point on $E$ away from $\left.\sum Q_{i}\right)$, then $\operatorname{deg} f=4$ and $f^{*}\left(\mathcal{O}_{\mathbb{P}^{1}}(1)\right) \simeq \mathcal{M}$. Since the quartic $\gamma\left(p_{i}\right)$ passes doubly through $Q_{i}$, we have $f\left(Q_{i}\right)=p_{i}$ for all $i$.

Case 2.2. If $\lambda$ has base points, then $\operatorname{deg} f \leq 3$. The base locus of $\lambda$ can contain at most $4-\operatorname{deg} f$ points from the set $\left\{Q_{i}\right\}$, hence $f\left(Q_{i}\right)=p_{i}$ holds for at least $4+\operatorname{deg} f$ values of $i$.

Now the previous lemma implies that either subcase is impossible for general choice of $p_{i}$, hence no such grove can exist. We have proved that $(3,3,2,8)$ is nondegenerate.

\section{Open PROBLEMS}

Whenever $\mathcal{A}$ is a finite set, we have the obvious enumerative problem of counting its cardinality. Beyond a handful of cases (see [23]) it is entirely open. In particular, I do not know the cardinality of $\mathcal{A}$ for $(2,4,4,10)$ or $(3,3,3,10)$.

It is also of interest to consider the family of positive dimensional schemes (with a fixed Hilbert polynomial) apolar to $\Lambda$. For instance, it is known that there are two twisted cubics apolar to a general web of quadrics in $\mathbb{P}^{3}$ (see [11, p. 32]). 
It is known that a general net of quadrics in $\mathbb{P}^{5}$ does not admit a polar octahedron (see 2]), contrary to what one would expect by counting parameters. However it is not known if such a net always admits an apolar rational normal quintic curve. A solution to this would help in elucidating the case $(5,2,3,8)$.

\section{REFERENCES}

[1] E. Arbarello, M. Cornalba, P. A. Griffiths, J. Harris. Geometry of Algebraic Curves, Volume I. Grundlehren der mathematischen Wissenschaften, No. 267. Springer-Verlag, New York, 1985.

[2] E. Carlini, J. Chipalkatti. On Waring's problem for several algebraic forms. Comment. Math. Helv., vol. 78, no. 3, pp. 494-517, 2003.

[3] C. Ciliberto, A. V. Geramita, F. Orecchia. Remarks on a theorem of HilbertBurch. Boll. Unione Mat. Ital. B(7). vol. 2, no. 3, pp. 463-483, 1988.

[4] C. Dionisi, C. Fontanari. Grassmann defectivity à la Terracini. To appear in Le Matematiche.

[5] I. Dolgachev. On certain families of elliptic curves in projective space. Ann. Mat. Pura Appl. (4), vol. 183, no. 3, pp. 317-331, 2004.

[6] I. Dolgachev. Dual homogeneous forms and varieties of sums of powers. Milan J. of Math. 72, pp. 163-187, 2004.

[7] I. Dolgachev, V. Kanev. Polar covariants of cubics and quartics. Adv. in Math., vol. 98, no. 2, pp. 216-301, 1993.

[8] R. Ehrenborg, G.-C. Rota. Apolarity and canonical forms for homogeneous polynomials. Europ. J. of Combinatorics, vol. 14, no. 3, pp. 157-181, 1993.

[9] D. Eisenbud. Commutative Algebra, with a View Toward Algebraic Geometry. Graduate Texts in Mathematics. Springer-Verlag, New York, 1995.

[10] D. Eisenbud, M. Green, J. Harris. Cayley-Bacharach theorems and conjectures. Bulletin of the A.M.S. (new series), vol. 33, no. 3, pp. 295-324, 1996.

[11] G. Ellingsrud, S.A. Strømme. The number of twisted cubic curves on a quintic threefold. Math. Scand., vol. 76, no. 1, pp. 5-34, 1995.

[12] C. Fontanari. On Waring's problem for many forms and Grassmann defective varieties. J. of Pure and Appl. Alg., vol. 174, no. 3, pp. 243-247, 2002.

[13] W. Fulton. Intersection Theory. Ergebnisse der Mathematik und ihrer Grenzgebiete, 3 Folge. Springer-Verlag, Berlin, 2nd edition, 1998.

[14] W. Fulton, J. Harris. Representation Theory, A First Course. Graduate Texts in Mathematics. Springer-Verlag, New York, 1991.

[15] A.V. Geramita. Inverse Systems of Fat Points. Queen's Papers in Pure and Applied Mathematics, Vol. X. Queen's University, 1995.

[16] P. A. Griffiths, J. Harris. Principles of Algebraic Geometry. Wiley Interscience, New York, 1978.

[17] J. Harris. Algebraic Geometry, A First Course. Graduate Texts in Mathematics. Springer-Verlag, New York, 1992. 
[18] R. Hartshorne. Algebraic Geometry. Graduate Texts in Mathematics. SpringerVerlag, New York, 1977.

[19] A. Iarrobino. Inverse system of a symbolic power II. The Waring problem for forms. J. of Algebra, vol. 174, no. 3, pp. 1091-1110, 1995.

[20] A. Iarrobino, V. Kanev. Power Sums, Gorenstein Algebras and Determinantal Loci. Springer Lecture Notes in Mathematics No. 1721, 1999.

[21] I. Lakatos. Proofs and Refutations. Cambridge University Press, 1976.

[22] F. London. Über die Polarfiguren der ebenen Curven dritter Ordnung. Math. Ann., vol. 36, pp. 535-584, 1890.

[23] K. Ranestad, F.-O. Schreyer. Varieties of sums of powers. J. Reine Angew. Math., band 525, pp. 147-181, 2000.

[24] B. Reichstein, Z. Reichstein. Surfaces parametrizing Waring presentation of smooth plane cubics. Mich. Math. J., vol. 40, pp. 95-118, 1993.

[25] T. G. Room. The Geometry of Determinantal Loci. Cambridge University Press, Cambridge, 1938.

[26] O. Schlesinger. Ueber die Verwerthung der $\vartheta$-Functionen. Math. Ann., vol. 31, pp. 183-219, 1888.

[27] A. Terracini. Sulla rappresentazione delle coppie di forme ternarie mediante somme di potenze di forme lineari. Annali di Matematica, Serie III, Tomo XXIV, 1915.

Jaydeep V. Chipalkatti

Department of Mathematics

University of Manitoba

Winnipeg, MB R3T 2N2, Canada.

email:chipalka@cc.umanitoba.ca 